\title{
Stability of PDF Controller with Stick-Slip Friction Device
}

\author{
Chih-Jung Huangף, Jia-Yush Yen§, Shu-Shung Lu†, \\ TGraduate Student $\S$ Associate Professor †Professor \\ Department of Mechanical Engineering, \\ National Taiwan University, Taipei, Taiwan, R.O.C. \\ email: jyen@ccms.ntu.edu.tw
}

\begin{abstract}
This paper presents the precision control of drive devices with significant stick-slip friction. The controller design follows the Pseudo-Derivative Feedback (PDF) control algorithm. Using the second order systern model, the PDF controller offers arbitrary pole placement. In this paper, the stability proof for the controller with stick-slip friction is presented. On the basis of this proof, the stability criteria are derived. The paper also includes computer simulation and experimental work to confirm theoretical results. The experiments conducted on a Traction Type Drive Device (TTDD) shows that control accuracy of as high as \pm 1 arc - second is achieved.
\end{abstract}

\section{Introduction}

Stick-slip friction is a commonly encountered phenomenon in mechanical systems. As described in $[1,6,10]$, stick-slip friction is the dominating factor that limits the performance of servo systems, and as stated in [10], this phenomenon has been the focus of many research efforts. During the past decade, many good results have been made available in the literature $[1], \ldots,[14]$.

The control of friction system is interesting not only because of its increased importance in the manufacturing industry but also because of the highly non-linear nature that makes it very difficult to be characterized. Actually, a significant portion of literature concerns either the modeling of the friction phenomenon or the identification of friction parameters $[3,4,5,6,11,14]$. Some of the reports presented very detailed observations on the friction force behavior; however, they are usually quite limited results due to the highly dependent nature of the friction phenomenon. Among the more popular works, the Bristle model of Haessig [11] captures the most detailed physical phenomenon, but is relatively numerically inefficient. The reset integrator model of Haessig and Karnopp [14] does not capture as much details, but is numerically more efficient.
The research efforts on active friction compensation are mainly available in the Robotic area $[6,8]$. Some of the research interests are also directed toward the machine tool applications [1]. The research interests then proceeds to the study of the direction compensation of the friction effect $[3,7,9,10,12]$. In most cases, the friction force are modeled as a constant disturbance. The control strategy is basically canceling the disturbance force. The theoretical analysis is simplified by assuming that the friction disturbance is canceled a-priori. Another very common approach is to use a Dither type excitation to eliminate the friction effect [8]. Their work covers mainly the experimental results. The development on active control involving stick-slip friction is more recent. Yang et al. [12] provided an adaptive version of the pulse width control. Theoretical analysis is also included. Since the parameters in the stick-slip friction model vary with many factors including load changes, lubrication conditions, and ambient temperature, ..., etc., many people apply adaptive techniques to the control problem $[2,12,13]$. The adaptive techniques are very effective at compensating for the slow varying friction force. It is also known to be sensitive to model structure mismatch. For low velocity friction compensation, the stick-slip friction becomes dominating. In this case more complicated control algorithms such as sliding mode controller [9], or two-degree-offreedom controller [7] must be used. Analytical works on the direct stick-slip friction compensation is very involved. Few studies are available. Tung, et al. in [7] mainly offered experimental results. [9] and [10] offered more theoretical results. Southward, et al. [10] offered a stability analysis using a cleaver Lyapunov function. Guzzella and Glattfelder [9] offered a stable controller using sliding mode approach. Both work$\mathrm{s}$ based on the cancellation of the non-linear friction force.

In this paper, the authors observed that the friction system is intrinsically stable. Even though Southward [10] and Karnopp [14] had both pointed out that stick-slip friction often results in system instability. This instability arises only due to improper excitation. In other words, the authors would like to address the question whether simple control algo- 
rithms can achieve satisfactory control or not. In this paper, we desire to investigate the performance of the Pseudo Derivative Feedback (PDF) control [15] for stick-slip friction drive. The PDF controller is used because it offers arbitrary pole placement for secondorder systems. In addition, the PDF controller is capable of disturbance rejection from the input side. To achieve low speed friction compensation, this paper adopts the Karnopp stick-slip friction model. A Lyapunov function for the control system stability is presented. The limit set for the PDF control is derived. The experimental results show that the PDF controller achieves positioning accuracy of as high as \pm 1 arc-second.

\section{Stick-Slip Friction Model}

The servo system under consideration contains a Traction Type Drive Device (TTDD) [16] with a servo motor as shown in figure 1. For the control purposes, the velocity response of the system is simplified by the commonly adopted first order system with stick-slip friction.

$$
\begin{aligned}
v_{r} & =\dot{\theta} \\
\dot{v}_{r}+a v_{r} & =\left(\tau-\tau_{f}\right)
\end{aligned}
$$

where $a$ is the system parameter, $\theta$ is the angular position, $v_{\tau}$ is the angular velocity, $\tau$ is the applied input, and $\tau_{f}$ represents the friction effect.

The model for the stick-slip friction $\tau_{f}$ is introduced by Karnopp [14], and is shown in figure 2.In Karnopp model, $m$ is the mass; $\sigma$ is the limiting angular velocity in the stick and slip regions; $\tau_{s t i c k}$ and $\tau_{s l i p}$ denote the stick and slip friction forces; $\tau_{h}^{+}$is the stick saturation friction of positive velocity; $\tau_{h}^{-}$is the stick saturation friction of negative velocity.

$$
\tau_{f}=\tau_{s l i d}\left(v_{r}\right)\left[\lambda\left(v_{r}\right)\right]+\tau_{s t i c k}(\tau)\left[1-\lambda\left(v_{r}\right)\right]
$$

In (2),

$$
\lambda\left(v_{r}\right)=\left\{\begin{array}{ll}
1, & \text { for }\left|v_{r}\right|>\sigma \\
0, & \text { for }\left|v_{r}\right| \leq \sigma
\end{array} \quad \sigma>0\right.
$$

The stick friction is represented by

$$
\tau_{\text {stick }}(\tau)=\left\{\begin{aligned}
\tau_{h}^{+}, & \text {for } \tau \geq \tau_{h}^{+}>0 \\
\tau, & \text { for } \tau_{h}^{-}<\tau<\tau_{h}^{+} \\
\tau_{h}^{-}, & \text {for } \tau \leq \tau_{h}^{-}<0
\end{aligned}\right.
$$

where $\tau_{s t i c k}(\tau)$ represents stick force of friction when $\left|v_{r}\right| \leq \sigma$. The positive and negative limits of the stick force are given by $\tau_{h}^{+}$and $\tau_{h}^{-}$. When $\left|v_{r}\right| \leq \sigma$ and the magnitude of the applied force is not larger than the limits of the stick force, the friction is equal to the applied force. The magnitudes of $\tau_{h}^{+}$and $\tau_{h}^{-}$in (4) are not presumed equal. The value of $\sigma$ is set to a small positive number only in the simulation to insure that the numerical integration algorithms remain stable.
The slip friction is modeled by

$$
\tau_{s l i p}\left(v_{r}\right)=\tau_{d}^{+}\left(v_{r}\right) U\left(v_{r}-\sigma\right)+\tau_{d}^{-}\left(v_{r}\right) U\left(-v_{r}-\sigma\right) \text {. }
$$

$U($.$) in (5) is the step function. The function \tau_{d}^{+}\left(v_{r}\right)$ is the slip friction for positive velocity, and the function $\tau_{d}^{-}\left(v_{\tau}\right)$ is the slip friction for negative velocity. The magnitudes of $\tau_{d}^{+}\left(v_{r}\right)$ and $\tau_{d}^{-}\left(v_{\tau}\right)$ are not presumed to be symmetric. Generally speaking, the magnitude of $\tau_{d}^{+}\left(v_{r}\right)$ is no greater than $\tau_{h}^{+}$; the magnitude of $\tau_{d}^{-}\left(v_{\tau}\right)$ is no greater than $\tau_{h}^{-}$. Here, assume that $\tau_{h}=$ $\max \left(\left|\tau_{h}^{+}\right|,\left|\tau_{h}^{-}\right|\right)=\left|\tau_{f}\right|$.

Altogether, equation (2) is used in the numerical simulation of $\tau_{f}$ as well as in the stability analysis.

\section{Controller design}

Consider the PDF control block diagram in figure 3. An additional anti-windup limiter is interduced to eliminate the reset windup phenomenon. Define the angular position $\theta=x_{2}$, the angular velocity $v=x_{3}$, and introduce an augmented state $x_{1}=\int \theta d t$. If one set the reference input $\Theta^{*}(s)$ to be 0 , it can be seen that PDF controller basically offers state feedback of the form

$$
\underline{K}=\left[\begin{array}{lll}
k_{i} & k_{p} & k_{d}
\end{array}\right] .
$$

With some algebra, it can also be shown that this system is completely controllable. Arbitrary pole placement is possible.

Notice that the friction effect, $\tau_{f}$, comes through a summing junction into the system input. Therefore, the friction force does not affect the close-loop poles. Using the PDF design technique to place the poles at $-r,-\alpha+\omega j$, and $-\alpha-\omega j$ where $r, \alpha$ and $\omega$ are positive reals. The resultant closed loop system becomes

$$
\begin{aligned}
& x_{1}=\int \theta d t \\
& x_{2}=\dot{x}_{1}=\theta \\
& x_{3}=\dot{x}_{2}=v_{\tau} \\
& \dot{x}_{3}=-a_{3} x_{1}-a_{2} x_{2}-a_{1} x_{3}-\tau_{f}
\end{aligned}
$$

where

$$
\left\{\begin{array}{l}
a_{1}=r+2 \alpha \\
a_{2}=2 \alpha r+\alpha^{2}+\omega^{2} \\
a_{3}=r\left(\alpha^{2}+\omega^{2}\right)
\end{array}\right.
$$

The state space representation for the closed-loop system is thus

$$
\begin{aligned}
& \underline{\dot{x}}=\underline{\mathrm{A} x}+\underline{\mathrm{B}} \tau_{f} \\
& y=\underline{\mathrm{x}}
\end{aligned}
$$

where

$$
\begin{gathered}
\underline{\mathrm{x}}=\left[\begin{array}{ccc}
x_{1} & x_{2} & x_{3}
\end{array}\right]^{\prime} \\
\mathrm{A}=\left[\begin{array}{ccc}
0 & 1 & 0 \\
0 & 0 & 1 \\
-a_{3} & -a_{2} & -a_{1}
\end{array}\right] \\
\underline{\mathrm{B}}=\left[\begin{array}{lll}
0 & 0 & -1
\end{array}\right]^{\prime} \\
\mathrm{C}=\left[\begin{array}{lll}
0 & 1 & 0
\end{array}\right]
\end{gathered}
$$




\section{Stability Proof}

Choose the following Lyapunov function candidate of the form.

$$
V(\underline{\mathrm{x}})=\underline{\mathrm{x}}^{\prime} \mathrm{P} \underline{\mathrm{x}}
$$

and

$$
\underline{\mathrm{P}}=\left(\begin{array}{lll}
p_{11} & p_{12} & p_{13} \\
p_{12} & p_{22} & p_{23} \\
p_{13} & p_{23} & p_{33}
\end{array}\right)
$$

Where $\underline{P}$ is a real symmetric positive definite matrix. From the well known Lyapunov stability theorem, Matrix A is a Hurwitz matrix, if and only if there exists some positive definite matrix $Q \in \mathcal{R}^{n \times n}$, such that

$$
\mathrm{A}^{\prime} \underline{\mathrm{P}}+\underline{\mathrm{PA}}=-\underline{\mathrm{Q}}
$$

has a corresponding unique solution for $\underline{\mathrm{P}}$, and $\underline{\mathrm{P}}$ is positive definite. The derivative of $V(\underline{\mathrm{x}})$ is given by

$$
\begin{aligned}
\dot{V}(\underline{\mathrm{x}}) & =-\mathrm{x}^{\prime} \mathrm{Q} \underline{\mathrm{x}}+2 \mathrm{X}^{\prime} \mathrm{P} \mathrm{B} \tau_{f} \\
& \leq-\underline{\underline{\mathrm{Q}}})|\underline{\mathrm{x}}|^{2}+2|\underline{\mathrm{x}}||\mathrm{PB}|\left|\tau_{f}\right| \\
& \leq-\underline{\sigma}(\underline{\mathrm{Q}})|\underline{\mathrm{x}}|\left(|\underline{\mathrm{x}}|-\left(2|\underline{\mathrm{PB}}| \tau_{h}\right) / \underline{\sigma}(\underline{\mathrm{Q}})\right)
\end{aligned}
$$

Assume $x_{r}=2|P B| \tau_{h} / \underline{\sigma}(\underline{\mathrm{Q}})$ and $\delta>0$, for all $|\underline{\mathrm{x}}|>$ $x_{r}+\delta$, than

$$
\begin{aligned}
\dot{V}(\mathrm{x}) & =-\underline{\sigma}(\underline{\mathrm{Q}})|\underline{\mathrm{x}}|\left(|\underline{\mathrm{x}}|-x_{r}\right) \\
& \leq-\underline{\mathrm{Q}})|\underline{\mathrm{x}}| \delta<0
\end{aligned}
$$

The system is bounded. The domain $B_{r}=\{\mathrm{x} \in$ $\left.\mathcal{R}^{3}:|\underline{x}| \leq x_{r}+\delta, \delta>0\right\}$ is a positive limit set and an invariant set. Define the distance $d(p, \Omega)$ is the distance between a point $p$ and a nonempty closed set $\Omega$. For all initial condition $\underline{x}_{0} \in \mathcal{R}^{3}, t_{0} \in \mathcal{R}_{+}$and trajectory $S\left(t, t_{0}, \mathrm{x}_{0}\right)$ of this system is satisfied that

$$
d\left(S\left(t, t_{0}, \underline{\mathrm{x}}_{0}\right), B_{r}\right) \longrightarrow 0 \text { as } t \longrightarrow \infty \text {. }
$$

And, given any $\varepsilon>0$ and $T<\infty$, there exists $a$ $t-t_{0} \geq T$ such that

$$
d\left(S\left(t, t_{0}, \underline{x}_{0}\right), B_{r}\right)<\varepsilon
$$

From above results, it satisfies the initial control specification that the state $x_{1}$ is bounded. On the other hand, the angular position state $x_{2}$ and the angular velocity state $x_{3}$ are also bounded.

From the above result, we can concentrate the focus on the final region for states $x_{2}$ and $x_{3}$. Consider the Lyapunov function candidate

$$
\mathrm{Q}=\left[\begin{array}{ccc}
0 & 0 & 0 \\
0 & q_{22} & q_{23} \\
0 & q_{23} & q_{33}
\end{array}\right]
$$

where $q_{11}=q_{12}=q_{13}=q_{21}=q_{31}=0$ means that the state $x_{1}$ is not considered. The solution of $\underline{P}$ is given by

$$
\underline{\mathbf{P}}=\left[\begin{array}{c}
\frac{a_{3}^{2} q_{33}+a_{1} a_{3} q_{22}}{2\left(a_{1} a_{2}-a_{3}\right)} \\
\frac{a_{3}\left(a_{2} q_{33}+q_{22}\right)}{2\left(a_{1} a_{2}-a_{3}\right)} \\
0
\end{array}\right.
$$

$$
\begin{aligned}
& \frac{a_{3}\left(a_{2} q_{33}+q_{22}\right)}{2\left(a_{1} a_{3}-a_{3}\right)} \\
& \frac{a_{3}\left(a_{1} q_{33}-2 q_{23}\right)+a_{2}^{2} q_{33}+a_{2}\left(q_{22}+2 a_{1} q_{23}\right)+a_{1}^{2} q_{22}}{2(3)} \\
& 2\left(a_{1} a_{2}-a_{3}\right) \\
& \frac{a_{3} q_{33}+a_{1} q_{22}}{2\left(a_{1} a_{2}-a_{3}\right)}
\end{aligned}
$$

It is straight forward to design the pole locations such that $\underline{P}$ is a positive definite matrix.

The derivative of $V(\underline{x})$ is

$$
\dot{V}(\underline{\mathrm{X}})=-q_{22} x_{2}^{2}-2 q_{23} x_{2} x_{3}-q_{33} x_{3}^{2}-2 p_{23} x_{2} \tau_{f}-p_{33} x_{3} \tau_{f}
$$

Let $q_{23}=\sqrt{q_{22} q_{33}}-\eta, \eta>0$. After some algebra the limit set of $x_{2}$ and $x_{3}$ as $t \rightarrow \infty$ can be derived as

$$
\begin{aligned}
& \left|x_{2}\right| \leq \frac{43933+a_{1} q_{22}}{\left(\alpha_{1} \alpha_{2}-4_{3}\right) q_{22}} \tau_{h}+\delta_{2} \quad \text { for some } \delta_{2}>0
\end{aligned}
$$

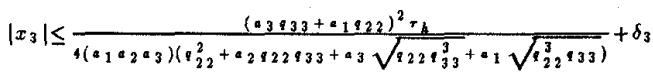

$$
\begin{aligned}
& \text { for some } \delta_{3}>0
\end{aligned}
$$

The positive parameters of $q_{22}$ and $q_{33}$ can be selected arbitrarily. Let $q_{22}=1$ and $q_{33} \longrightarrow \zeta$ for $\zeta>0$. One can use equation (18) and equation (19) to obtain

$$
\begin{aligned}
& \left|x_{2}\right| \leq \frac{(r+3 \alpha) r_{b}}{2\left(\alpha(\alpha+r)^{2}+\alpha \alpha^{2}\right)}+\delta_{2} \text { for } \delta_{2}>0 \text { as } t \rightarrow \rightarrow \infty \\
& \left|x_{3}\right| \leq \frac{(r+2 \alpha)^{2}+h}{8 \alpha\left(2 \alpha r+\alpha^{2}+\alpha^{2}+r^{2}\right)}+\delta_{3} \text { for } \delta_{3}>0 \text { as } t \rightarrow \rightarrow \infty
\end{aligned}
$$

Referring to equation (20) and (21), the larger the magnitudes of $r, \alpha$ and $\omega$, the smaller the positive limit set for the position and the velocity. On the other hand, positioning accuracy can be improved by increasing $r, \alpha$ or $\omega$. Notice that increasing $\omega$ produces high frequency input which is the same as using dither signals to remove the phenomena of stick-slip friction.

\section{Experiment Setup}

The experimental setup is shown in figure 4. The setup is based on a Traction Type Drive Device (TTDD). Conventional worm gear drives with only one worm experience large backlash, but the TTDD with two worms eliminates the backlash effect and isolates the friction problem [16].

Referring to figure 1, when the worm wheel rotates in the counterclockwise direction, worm 1 drives the worm wheel and act as the load worm. The torque clutch on worm 2 will force worm 2 to retain on the worm wheel, and acts as the control worm. As the worm wheel reverses its rotation, worm 1 and worm 2 switch functions. Worm 2 becomes the load worm, and worm 1 becomes the control worm. The assistant mechanism accomplishes this switching.

The position measurement uses a Canon $K \cdot 1$ super high resolution encoder. The angular velocity feedback comes from the position difference. The encoder produces 81,000 pulses/rev and achieves \pm 1 arc - second resolution. The encoder measures the worm wheel angle. A PC reads the pulses from 
the decoder and sends the voltage command through the DAC to the linear power amplifier that drives a Sanyo Denki U718T-012E DC servo motor.

\section{Simulation and Experimen- tal Results}

Consider the control block diagram in Figure 3. The identification is done by fitting the velocity response of the system into the ARMAX model in MATLAB. The resulted system transfer function is

$$
G_{p}(s)=\frac{\Theta(s)}{V(s)}=\frac{1}{s\left(I s+a^{\prime}\right)} .
$$

The parameters in (22) are $I=6.652 \times 10^{-6}$ and $a^{\prime}=1.656 \times 10^{-5}$. The parameters in the Karnopp model, figure 2 , are $\tau_{h}^{+}=0.78$ volt , the stick friction in the counterclockwise direction, $\tau_{d}^{+}=0.77 \mathrm{volt}$, the slip friction in the counterclockwise direction, $\tau_{h}^{-}=0.23$ volt, the stick friction in the clockwise direction, $\tau_{d}^{-}=0.22 v o l t$, the slip friction in the clockwise direction, and zero-region $\sigma$ in the Karnopp model equals $1 \times 10^{-5}$. These data are used for both the simulation and the controller design. For comparison purpose, the simulation and experimental results from PID controller will also be included.

The simulation results for the PDF control with various pole locations are shown in figure 5 . Limit cycle exits when the gains are set too low (placing the poles at $p=-15$ ). This is in agreement with the theoretical analysis. Overshoot does not occur with the PDF control in figure 5. The poles can be placed arbitrarily far left in the $s$-plane for the PDF control, because the simulation program does not consider signal noises and drive saturation.

The experimental results with the PDF control is shown in figure 6 . The results are the same as the simulation results for cases with poles placed at $p=-30$. Limit cycle appears again under the low gain situations ( $p=-15$ for example). For properly tuned system, the distance traveled does not affect the control result. Figure 7 shows small distance steppings for step sizes of 2 seconds, 4 seconds, and 6 seconds - It can be seen that even with very small movement (2 seconds) the control can still achieve satisfactory control with no steady state error.

\section{Conclusions}

This paper addressed the stability issue for the PDF control on the stick-slip friction system. The Karnop$p$ model is used for the stick-slip friction force. The Lyapunov stability for the system is presented. The limiting set for the PDF control is also derived. The size of the limiting set can be designed with the PDF controller parameters. Therefore, it is possible to improve the control accuracy through careful controller design.

The experiment setup is based on the TTDD. The TTDD eliminates the backlash by using two worms combined with two one-way clutches and two torque clutches. The TTDD achieve backlashless and selfretaining operation. Both the simulation results and experimental results show very good agreement with the theoretical analysis. With proper tuning, the PDF controller achieves positioning accuracy of as high as \pm 1 arc-second.

\section{References}

[1] Cetinkunt, S., Yu, W. L., and Donmez, A., 1994, "Friction Characterization Experiments on a Single Point Diamond Turning Machine Tool", Int. J. Mach. Tools Manufact, Vol.34, No.1, pp.19-32,1994

[2] Canudas, C., 1993, "Robust Control for ServoMechanisms Under Inexact Friction Compensation", Automatical, Vol.29,No.3,pp.757-761,1993.

[3] S.E.Mentzeloplou and B.Friedland "Experimental Evaluation $\mathrm{f}$ Friction Estimation and Compensation Techniques," 1994 American Control Conference, Baltimore, MD, Vol.3, pp. 3132-3136, 1994.

[4] Friedland and S.E. Mentzelopoulou "On Estimation of Dynamic Friction," 32nd IEEE Conference on Decision and Control, San Antonio, TX, Vol.2, pp. 1919-1924, 1993.

[5] Friedland and S.E. Mentzelopoulou and Y.J. Park "Friction Estimation in Multimass Systems," 1993 American Control Conference, San Franscisco, CA, Vol.2, pp. 1927-1931, 1993.

[6] Phillips, Stephen M., and Ballou, Kevin R, 1993, "Friction Modeling and Compensation for an Industrial Robot", Journal of Robotic Systems, Vol.10, No.7, pp.947-971,1993

[7] Tung, E. D., and Tomizuka, M., 1993, "Feedforward Tracking Controller Design Based on the Identification of Low Frequency Dynamics", Transactions of the ASME, Vol.115, SEP. 1993

[8] Yoshida, Yasuo., and Tanaka, Masato., 1993, "Position Control of a Flexible Arm Using a Dither Signal", Jsme International Joural, Series C, Vol.36, No.1,1993

[9] Guzzella, L., and Glattfelder, A. H., 1992, "Positioning of Stick-Slip Systems-Comparison of a Conventional and a Variable-Structure Controller Design", Proc. of 1992 ACC, Wp13, pp.1277-1281

[10] Southward, S. C., Radcliffe, C. J., and MacCluer, C. T., 1991, "Robust Nonlinear Stick-Slip Friction Compensation," Transactions of the ASME Journal of Dynamic Systems, Measurement, and Control, Vol.113, DECEMBER 1991, pp.639- 645.

[11] Haessig, Jr. D. A., and Friedland, B., 1991, "On the Modeling and Simulation of Friction" Transactions of the ASME Journal of Dynamic Systems, Measurement, and Control, Vol.113, SEPTEMBER 1991, pp.354-362

[12] Yang, S. and Tomizuka, M., 1988, "Adaptive Pulse Width Control for Precise Positioning Under the Influence of Stiction and Coulomb Friction," Transactions of the ASME Journal of Dynamic System $s$, Measurement, and Control, Vol.110,SEPTEMBER 1988, pp.221-227. 
[13] Canudas, C., Astrom, K. J. and Braun, K., 1986, "Adaptive Friction Compensation in DC Motor Drives", IEEE J. Rob. Auto., V.RA-3, n.6, Dec 1987, pp.681-685.

[14] Karnopp, D., 1985, "Computer Simulation of StickSlip Friction in Mechanical Dynamic Systems," Transactions of the ASME Journal of Dynamic Systems, Measurement, and Control, Vol.107,MARCH 1985 pp.100-103.

[15] Phelan, R. M., 1987, Feedback Control Systems (Privately Published Notes), Sibley School of Mechanical and Aerospace Engineering, Cornell University Press, Ithaca, NY

[16] Yang, T. H., 1993, "Douple Acting Type Dynamic Back Spacing Removed Driving System," US patent No.5265488.

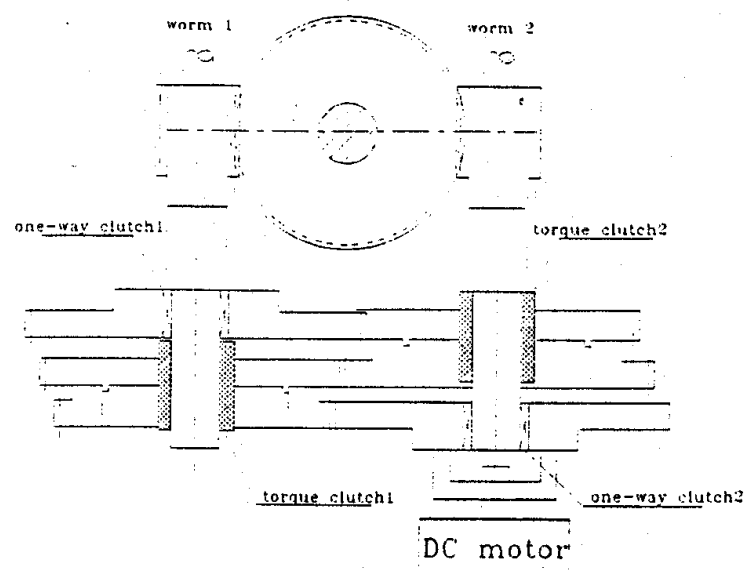

Figure 1: Traction Type Drive Device (TTDD)

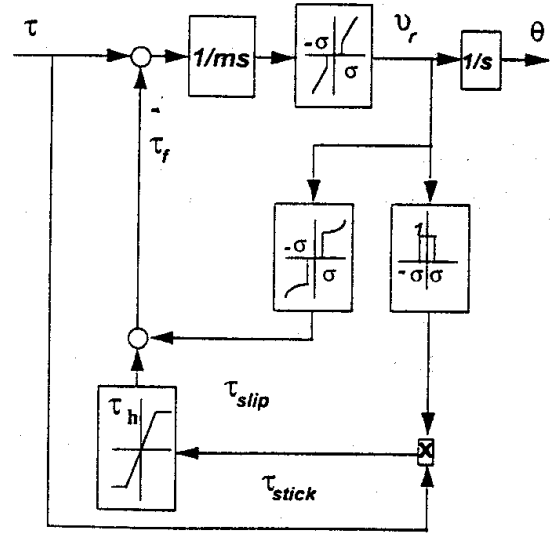

Figure 2: Karnopp model

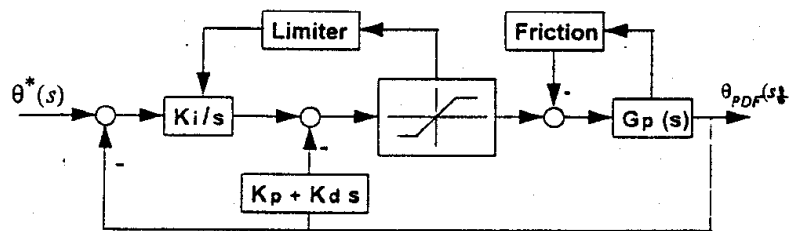

Figure 3: PDF Feedback Control Block Diagram

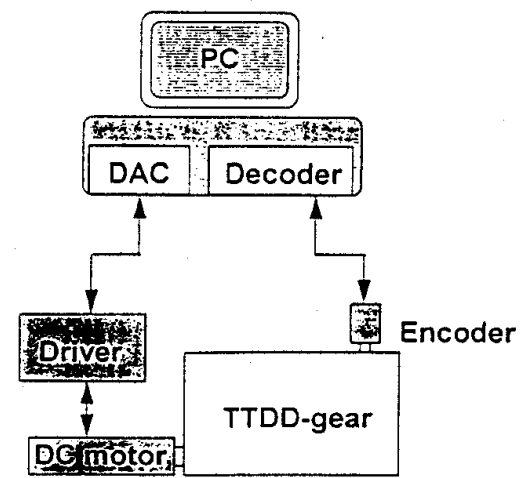

Figure 4: Stick-Slip Friction Experiment Setup output

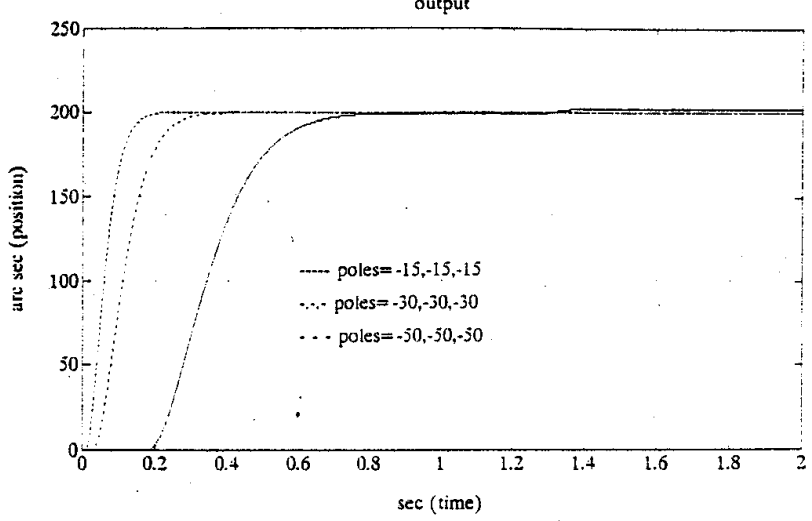

Figure 5: Simulation Results for PDF Control on Stick-Slip Friction System

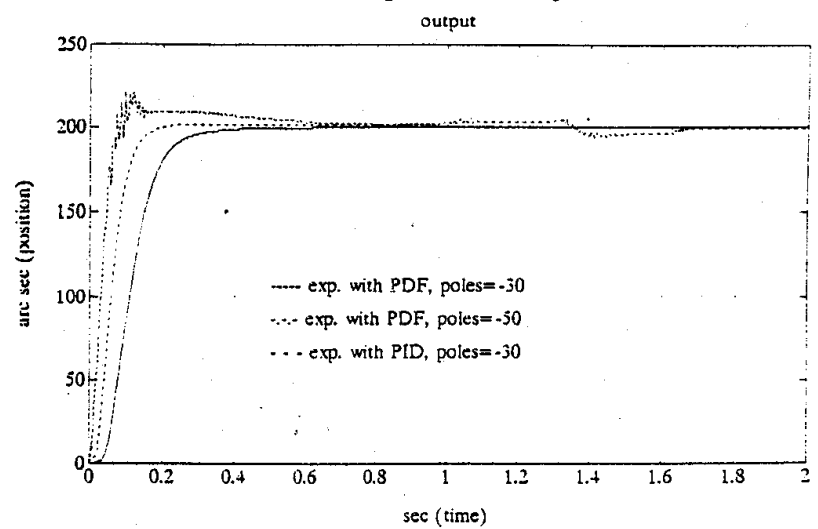

Figure 6: Experiment Results for PDF Control on Stick-Slip Friction System

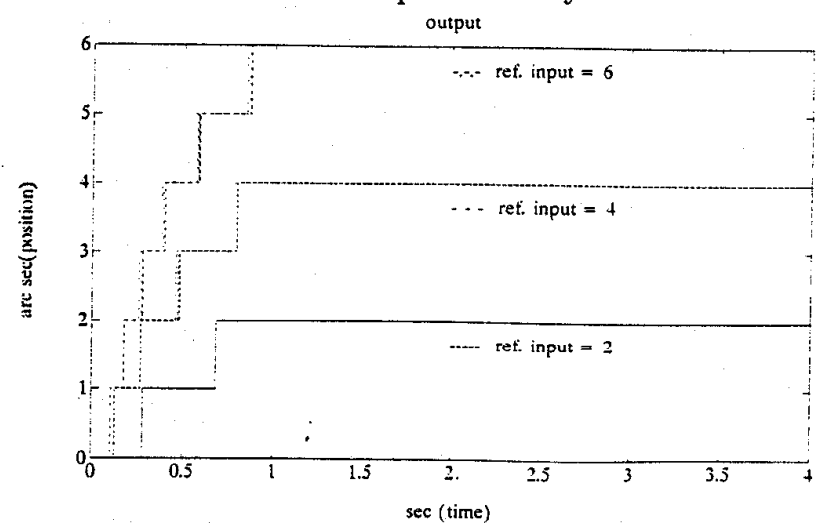

Figure 7: Small Steps PDF Control on Stick-Slip Friction System 